Meta

Journal des tradlucteurs

Translators' Journal

\title{
Entre traduction et normalisation terminologique
}

\section{Pierre Lerat}

Volume 35, numéro 4, décembre 1990

URI : https://id.erudit.org/iderudit/003471ar

DOI : https://doi.org/10.7202/003471ar

Aller au sommaire du numéro

Éditeur(s)

Les Presses de l'Université de Montréal

ISSN

0026-0452 (imprimé)

1492-1421 (numérique)

Découvrir la revue

Citer cette note

Lerat, P. (1990). Entre traduction et normalisation terminologique. Meta, 35(4),

798-801. https://doi.org/10.7202/003471ar d'utilisation que vous pouvez consulter en ligne.

https://apropos.erudit.org/fr/usagers/politique-dutilisation/ 


\section{ENTRE TRADUCTION ET NORMALISATION TERMINOLOGIQUE}

L'Institut national de la langue française est un laboratoire du CNRS dont les travaux sont consacrés aux vocabulaires français. L'une de ses composantes s'appelle Trésor général des langues et parlers français. Placée sous la responsabilité directe du directeur de I'INALF, Bernard Quemada, cette unite de recherche linguistique $I$ est spécialisée dans les bases de données (textuelles, dictionnairiques bibliographiques et néologiques). Elle comprend une section à Nancy (la principale, celle qui gère notamment la banque de données textuelles FRANTEXT, la bibliographie analytique de la linguistique française et la mise à jour des fonds textuels et lexicaux), une à Besançon (qui édite les «datations et documents lexicographiques») et une à Paris-Villetaneuse (où sont élaborées notamment les «archives du français contemporain» qui alimentent en néologismes de la langue ordinaire le dictionnaire du Trésor de la langue française).

C'est de cette URL1 que relève le Centre de terminologie et de néologie créé en septembre 1987. Sa place parmi les organismes responsables de la terminologie en France, ses orientations scientifiques et ses différentes fonctions officielles sont décrites dans le numéro de septembre de La banque des mots, où le lecteur pourra trouver une information plus ample. Ce qu'il a paru utile de faire ici est sensiblement différent: il s'agit de signaler aux traducteurs, aux spécialistes de l'aménagement linguistique en France ou ailleurs, aux documentalistes et aux enseignants de langues, de sciences ou de techniques ce qu'ils peuvent raisonnablement attendre de cet organisme original à maint égard.

En quoi un centre spécialisé dans le français, relevant d'un appareil scientifique national et de création aussi récente peut-il favoriser la traduction, répondre à des besoins pratiques et tirer parti d'expériences qui ne sont pas les siennes? Ces questions méritent des réponses précises. Celles que vise à fournir cet article ne sont pas programmatiques mais mettent en perspective des travaux à court, moyen et long terme dont les premiers bénéficieront d'une publication dès 1989 .

Mon espoir est bien entendu d'être lu jusqu'an point final, d'où un effort de concision. Mais pour que le lecteur particulièrement pressé puisse juger d'emblée de la pertinence de nos choix, voici en résumé la philosophie du CTN:

- partir d'une bonne description linguistique et notionnelle des termes est nécessaire pour faire de la terminologie adéquatement, c'est-à-dire utilement, à plus forte raison si la visée est bilingue ou multilingue;

- la terminologie de demain sera faite de fichiers de domaines particuliers bien balisés, conçus en fonction d'exploitations multiples, à commencer par les transferts de connaissances sous toutes leurs formes, où les progrès technologiques ne dépendront ni de l'expertise du spécialiste du domaine ni de la prise en compte des variables non mécaniques de l'acceptabilité des mots :

- elle est à la portée de traducteurs individuels bien formés, de petites entreprises, d'équipes universitaires et d'administrations, grâce à la banalisation de la micro-informatique, à laquelle nous sommes reconnaissants de deux avantages considérables : un coût non prohibitif et une autonomie totale dans le travail.

Comme on le verra, ces orientations ne sont pas des vues de l'esprit mais des réponses à une attente des pouvoirs publics (première partie), à celle du CNRS et des étudiants en formation (deuxième partie), ainsi qu'au marché français et francophone (troisième partie) et au besoin d'un partage réaliste des tâches dans un secteur d'activité où il $y$ a beaucoup à faire (quatrième partie).

\section{UN ORGANISME RÉCENT}

La création récente du CTN lui donne une place particulière dans un dispositif français caractérisé principalement par l'existence, depuis une quinzaine d'années, de trois institutions clairement finalisées: le Conseil international de la langue française (CILF), qui publie des dictionnaires, des ouvrages et aussi l'unique revue française de terminologie ( $L a$ banque des mots), l'Association française de terminologie (AFNOR), qui a constitué à partir des normes industrielles une banque de données terminologiques (NORMATERM), et enfin les Commissions ministérielles de terminologie, dont les listes sont consultables sur trois supports (le Journal officiel, des ouvrages et le minitel). Les fonctions normatives étant prises en charge par les deux dernières institutions, le CTN est en mesure de se consacrer à la description, à la documentation et à la méthodologie. Pour les publications, l'expérience acquise par le CILF a conduit le CTN à s'entendre avec lui pour y éditer son premier vocabulaire, $L e$ 
Dictionnaire français des organisations panafricaines (DIFOP), à paraître au printemps de 1989, sous la responsabilité de $\mathrm{M}$. Kalonji. De même, plutôt que de créer une revue concurrente de La banque des mots, il a paru préférable de prendre en charge un numéro par an de ce périodique (à partir de septembre 1988).

Un autre avantage de la jeunesse est l'absence d'inhibition face aux grandes réalisations terminologiques. Contemporains du développement de la micro-informatique, nous élaborons des fichiers qui n'ont en commun que la structure de chaque fiche. Le premier, qui a été entrepris dès l'ouverture du Centre, en septembre 1987, a été un banc d'essai destiné à l'acquisition d'un savoir-faire. Il s'agit d'une terminologie de la robotique, thème dont le choix ne nous appartenait pas puisqu'il résulte d'engagements antérieurs de la France dans le programme de collaboration au sein du Réseau international de néologie et de terminologie (RINT). Composite, en partie de seconde main, mais menée à bien et consultable sous forme de listages, elle a joué un rôle d'autoformation au contact des spécialistes du domaine, notamment à l'AFNOR. Les leçons de l'expérience sont actuellement réinvesties dans une base de données terminologiques sur l'intelligence artificielle, d'où sera puisé sélectivement, dès 1989 , un Dictionnaire de l'intelligence artificielle, grâce à un contrat avec EC2, spécialiste de l'LA.

La télématique rend disponibles désormais des ressources terminologiques accumulées par la BTQ (Banque de terminologie du Québec) et EURODICAUTOM (banque de terminologie des communautés européennes), et celles de TERMIUM (banque de terminologie du Canada) sont sur CD ROM. L'heure n'est donc plus à de nouvelles grandes entreprises visant l'exhaustivité, mais aux échanges de donnés et de services, aux innovations techniques et méthodologiques, aux transferts de connaissances. Avec un PC-AT milieu de gamme et un système de gestion de bases de données de la famille de DBASE PLUS, la preuve est faite que beaucoup de fonctionnalités sont accessibles à un prix non prohibitif. La concurrence des logiciels peut dans ces conditions jouer au sein d'une même équipe: c'est ainsi que notre ingénieur informaticien, Roland Dachelet, secrétaire de l'Association pour la recherche cognitive (ARC), a entrepris une évaluation comparative de FOXBASE + et de MICROCEZEAU 4. Le temps des grandes évidences est révolu en terminologie comme en traduction assistée, en lexicographie et en documentation. C'est du moins cette conviction qui nous a conduits à organiser pour janvier 1989 un colloque «Terminologie et industries de la langue», où les stands devraient avoir autant d'importance que les communications de scientifiques, de fabricants et d'usagers.

\section{UNE ÉQUIPE DE RECHERCHE APPLIQUÉE}

L'appartenance à un grand organisme scientifique impose bien entendu une forte part de recherche fondamentale. Dans le cas présent, les travaux de thèses en cours donnent une idée de l'ampleur du champ à couvrir: syntaxe des unités terminologiques complexes, morphologie des mots savants, relations de sens, part des emprunts dans les termes récents, repérage des éléments formants dans les fichiers de termes et dans des discours spécialisés. Hors d'un laboratoire, la masse des données observables est moins accessible, le travail d'équipe difficile, l'information dispersé; il est donc temps que le CNRS prenne sa place dans cette discipline carrefour. Mais il faut bien voir que rien n'est gratuit dans les préoccupations énumérées ci-dessus : la morphologie est le fondement objectif du «génie de la langue», la sémantique est le moyen de «naviguer» de concept à concept dans une base, et la connaissance des emprunts scientifiques et techniques au jour le jour est la seule façon de prendre la mesure de l'internationalisation des échanges verbaux et de garantir une bonne adéquation des moyens d'action dont disposent les décideurs politiques.

Mais il n'y a pas de recherche appliquée sans production. C'est pourquoi le CTN non seulement programme des dictionnaires imprimés mais se propose de répondre à un récent appel d'offres des communautés européennes et à une commande de contribution à un CD ROM. Cette soumission aux règles du marché aurait pu naguère surprendre de la part du CNRS mais la «valorisation» est désormais non seulement tolérée mais encouragée.

Ce va-et-vient entre la théorie linguistique et la pratique la plus professionnelle est favorisé par trois activités qui sont classiques dans beaucoup de laboratoires: une documentation systématique, des échanges avec la communauté internationale, une contribution à la formation. Un fonds national de terminologie se constitue progressivement, en ajoutant aux ressources accumulées par des organismes antérieurs, notamment AFTERM et FRANTERM, les revues, les dictionnaires et les ouvrages de base. Les échanges de doubles avec des partenaires limitent les coûts; de même, les informations fournies à des stagiaires le sont à titre gratuit, mais contre un engagement de fournir au CTN un exemplaire du travail qui en aura bénéficié.

La formation est assurée au niveau universitaire exclusivement, dans le cadre du doctorat de sciences du langage de Paris III - Paris XIII. L'orientation «linguistique» en limite l'accès aux titulaires d'une maîtrise de langue ou de linguistique (ou d'un diplôme équivalent); l'orientation «appliquée» induit des traductions d'articles, des minifichiers de termes et des exposés-discussions, ainsi que des conférences de professionnels. Le fort pourcentage des étrangers est par lui-même enrichissant quand il s'agit d'un domaine comme celui-ci, où le degré de développement économique, les politiques linguistiques et les techniques sont des variables aussi importantes que les structures des langues.

Les échanges internationaux sont encore peu actifs, pour des raisons qui tiennent à des retards dont nous ne sommes pas les seules victimes; la lenteur de la mise en place des réseaux à caractère politique, le 
manque de conventions bilatérales entre organismes techniques et une circulation insuffisante des données et des personnes sont des inconvénients auxquels il est possible de remédier grâce à deux moyens au moins: les solidarités de voisinage (comme entre le CTN et l'Union latine) et la formation (le boursier étranger d'aujourd'hui est le collègue de demain).

\section{TERMINOLOGIE ET NÉOLOGIE}

La priorité accordée au français résulte d'une situation géo-historique particulière (et elles le sont toutes, ce qu'on oublie trop souvent en matière de politique linguistique). La terminologie s'est développée au $\mathrm{XX}^{\mathrm{e}}$ siècle dans des conditions variées. Sa justification principale en URSS est bien connue: c'est une contribution à la gestion du multilinguisme; il en va de même aux communautés européennes. Une deuxième stimulation, qui a joué tout particulièrement en Autriche sous l'impulsion de Wüster, et qui a également contribué à l'essor de la terminologie soviétique, est la normalisation terminologique comme facteur du progrès scientifique et technique; elle est compatible avec l'unilinguisme, et se trouve même favorisée par la considération d'un système linguistique unique. Un troisième cas est le besoin de tout dire dans deux langues concurrentes; tel est le moteur des entreprises à grande échelle qu'ont suscitées les pouvoirs publics au Canada (au Québec notamment) et celui des efforts déployés en Belgique ou en Tunisie - pour ne prendre d'exemples que dans des pays où le français est l'une des langues dominantes. Où se situe la France dans cette typologie?

À la marge, jusqu'à ce jour, du fait de la tradition de gallocentrisme dont Rivarol est la figure archétypique et dont la remise en question trop tardive sous la pression des échanges économiques suscite actuellement une insécurité linguistique par rapport à laquelle la querelle du franglais, si bien remise à sa juste place par le regretté Pierre Trescases, apparaît comme un simple signe avant-coureur des vrais enjeux: la mondialisation des marchés (bien avant l'«Europe de 1992»), le transfert des connaissances, la technicisation de ces dernières. Certes, l'intérêt des ingénieurs pour la normalisation n'est pas nouveau, non plus que le souci d'une partie des hauts fonctionnaires de limiter autant que possible l'anglicisation dans les limites des compétences des ministères où ils font une partie de leur carrière, mais pendant que les uns et les autres se penchent sur les quelques dizaines de termes qui sont de leur ressort, les «observatoires» de l'INALF enregistrent depuis des années des néologismes par milliers. Encore ne s'agitil que de termes techniques plus ou moins banalisés (attestés dans la grande presse) : autant dire que ces mots ne sont que la partie la plus visible d'une néologie active dans les milieux de la production intellectuelle et matérielle. Il est donc clair que l'extension de l'observation à des corpus spécialisés s'imposait. Il faut bien voir aussi, en même temps, que la connaissance est la condition nécessaire mais non suffisante d'une politique linguistique adaptée, où la formation scolaire et professionnelle et l'adhésion du tissu social (principalement dans les entreprises) sont plus faciles dès lors que les langues étrangères sont elles aussi prises au sérieux.

A mon avis, il faut agir selon les trois modèles: le multilinguisme pour les échanges de toutes sortes dans le vaste monde, le bilinguisme pour les secteurs à haut risque linguistique (secteurs de pointe), l'harmonisation au sein de la langue française, menacée de se différencier exagérément en technolectes et en géolectes. On peut évidemment partir des besoins ressentis en traduction: c'est ce qui se fait en France dans les multinationales, les grandes entreprises exportatrices et les établissements d'enseignement supérieur (écoles d'interprètes-traducteurs, maîtrises de langues étrangères appliquées, etc.). La stratégie du CTN ne fait en aucune façon double emploi avec ces partenaires potentiels, car son centre de gravité est le français: dans ses fichiers cette langue est le coeur, l'anglais la première couche enveloppante, les autres grandes langues du monde les cibles escomptées à moyen terme. Quand je suis à mon bureau, au premier plan se trouve du mobilier ayant appartenu à FRANTERM, association dont l'effort de publication a été consacré, utilement mais dans les limites d'une action où la priorité était la lutte contre le franglais dans l'administration, à des recueils de néologismes officiels; mais au second plan, il y a un calendrier chinois, cadeau d'une étudiante... et personne d'entre nous n'est encore en mesure de le lire. Voilà nos racines et notre horizon.

Bien entendu, les trois types d'opérations ne peuvent pas être échelonnés dans le temps, du fait de l'égalité des urgences. Quelques exemples montreront d'ailleurs des interconnexions. Ainsi, DIFOP est un dictionnaire unilingue, mais, le cas échéant, il sera techniquement facile à traduire dans une langue d'Afrique. Le dictionnaire de l'intelligence artificielle est un faux bilingue (avec seulement, pour l'anglais, les équivalents), mais sa conception systématique se prête à tous les compléments concernant l'anglais ou telle autre langue que l'on voudra. Il ne s'agit que de ciblage, l'essentiel est de pouvoir faire face à la demande grâce à des collaborations: de même qu'il faut faire appel à des experts du domaine, il est capital de recourir à des experts de langues, à commencer par les locuteurs natifs. Mais le cœur du travail terminologique est la gestion de fichiers de termes et de notions en prévision d'un faisceau maximal d'utilisations.

Cette visée multifonctionnelle se reflète dans la nature des informations prises en compte:

- pour les champs documentaires, outre les sources, les domaines, etc., des indications sur la combinatoire, les éléments constitutifs et les standards officiels;

- pour les champs logiques, des relations utilisées en intelligence artificielle ;

- pour les champs chronologiques, non seulement la date des énoncés utilisés et celle de la mise à jour de la fiche (ou, par défaut, de l'enregistrement), mais aussi l'année de la première utilisation connue du terme. 
Ces trois séries d'indications sont conformes à la politique scientifique de l'INALF, et plus spécialement à l'entreprise d'informatisation du Trésor de la langue française. La dernière va dans la même direction que BORNEO (banque ordonnée de néologismes de la langue générale) et que les DDL (datations et documents lexicographiques); on voit aussi qu'avec le temps il deviendra possible de contribuer utilement a l'histoire des terminologies, donc à celle des sciences et des techniques elles-mêmes.

Dans ces conditions, il est inutile d'insister longuement sur le caractère complémentaire des deux notions de «terminologie» et de «néologie»: en terminologie l'étude des vocabulaires des domaines récents fait apparaître des néologismes, en néologie les concepts nouveaux invitent à des investigations dans des domaines de connaissances évolutifs, qui sont des gisements terminologiques. D'où notre décision de donner exactement la même structure aux fichiers néologiques et aux fichiers terminologiques.

\section{ANALYSE LINGUISTIQUE, \\ DOCUMENTATION ET HARMONISATION}

La bonne traduction et la normalisation non autoritaire ont en commun d'exiger trois préalables: une analyse correcte des faits de langue, une documentation systématique, une harmonisation des données.

Le premier point a été recommandé par $\mathrm{B}$. Quemada dès le temps du Haut comité de la langue française, dont il était membre, mais le rôle de linguiste en la matière a nécessairement quelque chose de répétitif car il faut toujours rappeler aux nonspécialistes des évidences comme celles-ci : la dénomination n'est pas la paraphrase, les sigles ne se décrètent pas, la structure d'une langue est évolutive, sa syntagmatique doit être respectée, le meilleur terme est celui qui concilie l'intercommunication dans le monde avec l'kesprit de clocher» au sens de Saussure, etc.

La documentation systématique est également une exigence d'information fiable, de rapidité d'accès aux sources et aussi de codage lisible par des machines à l'intention d'usagers humains. L'informatique peut être utilisée de façon maximaliste (traduction automatique) ou minimaliste (l' «équivalent» presse-bouton). Entre les deux, l'assistance à la traduction humaine intelligente passe par l'information sur les liens logiques, la définition, la combinatoire et l'autorité relative des termes concurrents.

L'harmonisation est la seule façon d'éviter à la fois la babélisation, la créolisation et le fossé entre le droit et le fait. Elle suppose l'information réciproque des gestionnaires de bases de données, la standardisation des méthodes de travail en terminologie à l'échelle d'une langue donnée et au niveau mondial, ainsi que la mise à la disposition des usagers de données sélectionnées et fiables. En somme, beaucoup de travail en perspective, avec beaucoup de partenaires.

PIERRE LERAT Centre de terminologie et de néologie, Paris, France 